\title{
A giant coronary artery aneurysm and recurrent ST-segment elevation myocardial infarction: A management dilemna
}

\author{
Benjamin Seguy ${ }^{1}$, Eric A. Osborn ${ }^{2}$, Mathieu Pernot ${ }^{3}$, Edouard Gerbaud ${ }^{1}$ \\ ${ }^{1}$ Intensive Cardiology Care Unit - Interventional Cardiology, Hôpital Cardiologique \\ du Haut Lévêque, Bordeaux University Hospital, Pessac, France \\ ${ }^{2}$ Cardiology Division, Beth Israel Deaconess Medical Center, Harvard Medical School, Boston, MA, USA \\ ${ }^{3}$ Medico-Surgical Department (Valvulopathies, Cardiac Surgery, Adult Structural Interventional \\ Cardiology), Hôpital Cardiologique du Haut-Lévêque, Bordeaux University Hospital, Pessac, France
}

Giant coronary artery aneurysms (CAA), generally described as dilation that exceeds the reference vessel diameter by 4 times [1,2], are a rare phenomenon and therefore the optimal management strategy in these clinical scenarios remains controversial without available evidencebased guidelines. CAA treatment options may include conservative medical therapy, percutaneous coronary intervention (PCI), and surgical revascularization [2]. Herein, is described the dilemma of managing an acute occlusion of a proximal left anterior descending artery (LAD) giant CAA, where each of the described treatment strategies were utilized in succession, ultimately leading to successful surgical intervention after the failure of less invasive medical and percutaneous methods.

A 50-year-old male was referred to the documented department after presenting high lateral ST-segment elevation myocardial infarction (STEMI). Coronary angiography revealed diffuse atherosclerosis and aneurysmal coronary arteries, notable for a giant proximal LAD coronary aneurysm with a hazy filling defect at the distal aneurysm edge and Thrombolysis in Myocardial Infarction (TIMI) grade 2 flow (Suppl. Movie 1). Left ventriculography confirmed the presence of an anterolateral wall motion abnormality (calculated left ventricular ejection fraction [LVEF] of 50\%). Due to resolution of patient symptoms in the catheterization laboratory, in the context of angiographic findings, intervention was deferred in favor of a conservative treatment strategy. The patient was treated with triple anti-thrombotic therapy including acetylsalicylic acid (ASA), clopidogrel, and warfarin with a goal international normalized ratio (INR) of 2.0-3.0. Four months after discharge, warfarin was switched to rivaroxaban (20 mg per day) because of persistent subtherapeutic INR levels.

The patient remained clinically well until 10 months after his initial presentation, when he developed sudden-onset recurrent chest pain. Initial electrocardiogram (ECG) revealed an acute anterior STEMI. Emergency coronary angiography was performed within $6 \mathrm{~h}$ of symptom onset and demonstrated thrombotic occlusion of the proximal LAD inside the aneurysm (Fig. 1A). Left ventriculography showed a new, severely reduced ejection fraction (calculated LVEF of 28\%) with marked hypokinesis of the anterior segments. An urgent Heart Team meeting between interventional cardiology and cardiac surgery was assembled, and a consensus decision was to proceed with primary angioplasty in the context of STEMI. Bolus IV heparin was given and the activated clotting time remained at $>350 \mathrm{~s}$ throughout the case indicating adequate systemic anticoagulation. Following engagement of the left main coronary artery with a 6 French EBU 4.0 guiding catheter, an intracoronary abciximab bolus $(0.25 \mathrm{mg} / \mathrm{kg}$ body weight $)$ was immediately administered. The LAD occlusion was crossed using a Sion blue wire (Asahi Intecc, Nagoya, Japan) with difficulty due to the aneurysmal sac. Primary angioplasty at the site of occlusion with a compliant balloon (Ryujin ${ }^{\mathrm{TM}}$

Address for correspondence: Edouard Gerbaud, MD, PhD, Soins Intensifs Cardiologiques — Plateau de Cardiologie Interventionnelle, CHU de Bordeaux, Hôpital du Haut Lévêque, 5 avenue Magellan, 33604 Pessac, France, tel: +33 557675 536, fax: +33 557656 316, e-mail: edouard.gerbaud@chu-bordeaux.fr 


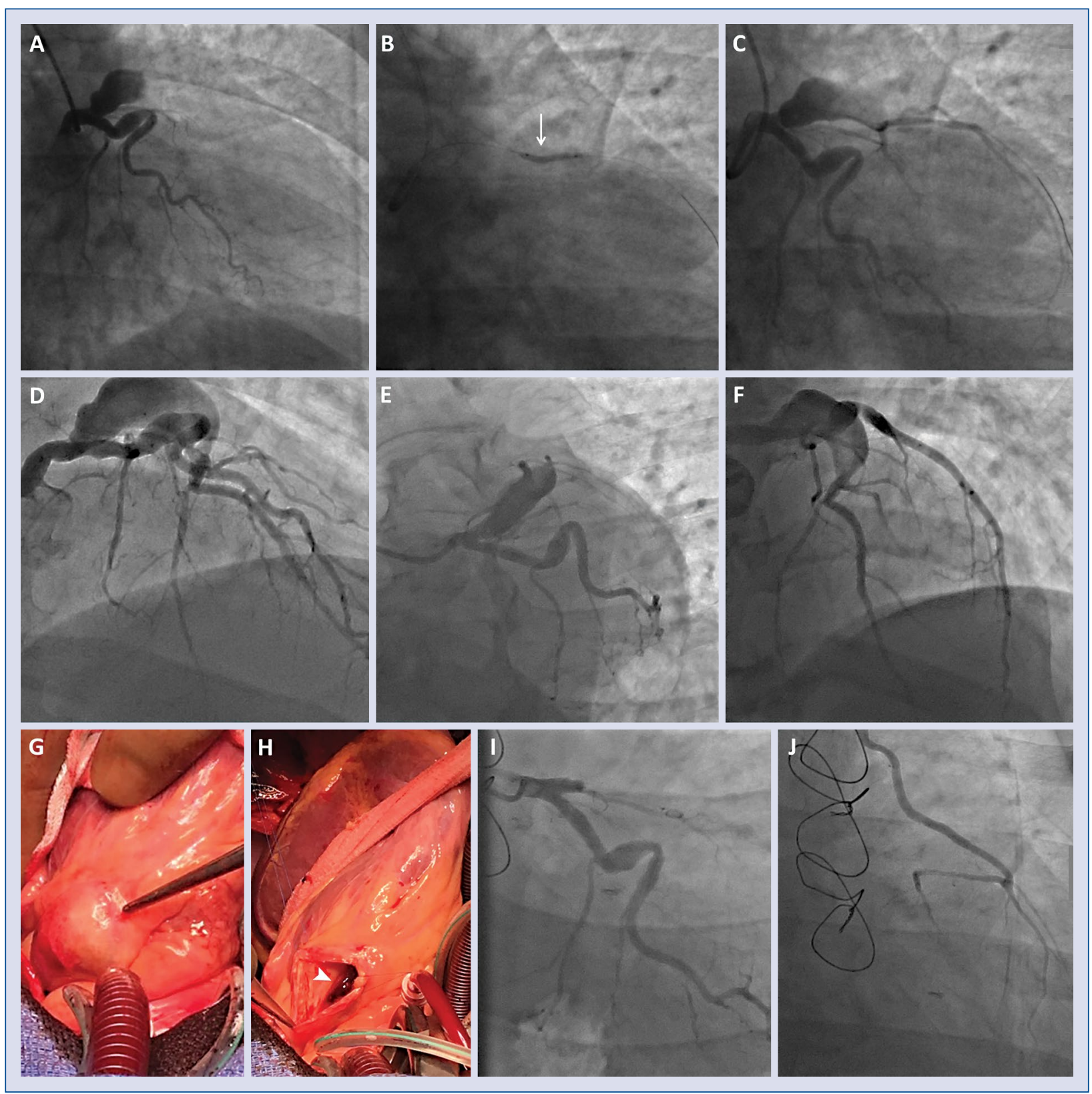

Figure 1. Emergent coronary angiography demonstrated occlusion of the proximal left anterior descending artery (LAD) inside the aneurysm (A). The balloon inflation clearly revealed the distal funnel of the aneurysm: there was a $20^{\circ}$ angle (arrow) between the intra-aneurysm portion and the extra-aneurysm portion of the balloon inside the LAD (B). Primary angioplasty at the site of occlusion restored Thrombolysis in Myocardial Infarction (TIMI) grade 2 flow into the distal LAD (C). Six days later, a repeat coronary angiography confirmed partial regression of the thrombus in a patent LAD with TIMI grade 3 flow (D, E, F). Then, the Heart Team decision was to proceed with cardiothoracic surgery combining aneurysm exclusion and coronary artery bypass graft. During surgery the aneurysm was opened showing residual thrombus inside (arrowhead; G, H). Control coronary angiography revealed a total exclusion of the aneurysm (I) and final good result of the left internal mammary artery bypass grafting (J).

Plus, $2.0 \times 15 \mathrm{~mm}$, Terumo, Japan) restored TIMI grade 2 flow into the distal LAD. Of note, during coronary balloon inflation the distal funnel of the aneurysm was exposed, with a calculated $20^{\circ}$ angle between the intra- and extra-aneurysm portions of the balloon inside the LAD (Fig. 1B, C). At this point, as the patient's symptoms had resolved in the catheterization laboratory following successful balloon angioplasty and, given angiographic findings showing a large thrombus burden inside 
the aneurysm, the treatment plan was to continue medical therapy followed by a re-look angiography 5-7 days later. Dual antiplatelet therapy (ASA and clopidogrel) was continued, in addition to anticoagulant therapy with IV unfractionated heparin. The patient remained stable on medical therapy in the Intensive Cardiac Care Unit, and underwent repeat coronary angiography 6 days after primary angioplasty demonstrating partial regression of the thrombus in a patent LAD with TIMI grade 3 flow (Fig. 1D-F). The maximal diameter of the aneurysm exceeded $15.0 \mathrm{~mm}$ associated with a sac length of $60 \mathrm{~mm}$ by intravascular ultrasound imaging. Based on the size, the aneurysm did not appear amenable to PCI with commercially available polytetrafluoroethylene (PTFE)-covered stents. Thus, in consultation with the Heart Team, a surgical approach combining aneurysm exclusion and coronary artery bypass grafting (CABG) was recommended. On day 21 , the patient underwent an uncomplicated on-pump single vessel CABG with bypass of the LAD using the left internal mammary artery (LIMA). Intraprocedurally, the LAD coronary aneurysm (Fig. $1 \mathrm{G}, \mathrm{H}$ ) was released after transection of the pulmonary artery. The aneurysm was then opened and the proximal and the distal funnels were closed using a venous patch. Control coronary angiography revealed a total exclusion of the aneurysm (Fig. 1I) and good result of the LIMA bypass grafting (Fig. 1J). At 1-year follow-up, the patient remains asymptomatic with a stable ECG and LVEF of $38 \%$ assessed by cardiac magnetic resonance imaging.

In asymptomatic or stabilized patients with CAA thrombosis, several case reports have highlighted various successful conservative treatment strategies [2,3]. At present, the role of direct oral anticoagulants for long-term treatment of CAA is currently unknown. Medically managed patients likely require lifelong therapy, as they are potentially at high risk for recurrent thrombosis should anti-thrombotic treatment be interrupted [2]. However, in patients presenting with STEMI, emergent PCI should be considered [4], optimally in the context of a Heart Team decision-making approach. Percutaneous exclusion of the CAA with one or many overlapped PTFE-covered stents may be of value in select patients with "short" aneurysms that are less than $10 \mathrm{~mm}$ in diameter located in straight, large proximal vessels $[5,6]$. Furthermore, anatomical suitability should be considered carefully before covered stent use, as many of these devices require large, supportive guides and guidewires among other techniques to enhance delivery [7]. Recently, Boi et al. [8] reported a promising technique consisting of two different layers of overlapping stents named "double-layer bridging". Use of spirals to obtain aneurysm thrombosis is sometimes useful [9]. Patients presenting with CAA involving the left main coronary artery, bifurcation of significant side branch vessel lesions, multivessel coronary artery disease, giant aneurysms [10], or a separate indication for cardiothoracic surgery unrelated to CAA are usually referred for surgical revascularization. Although this case report does not correspond exactly to these criteria, the present Heart Team considered that surgical revascularization was the most appropriate strategy for favorable long-term outcome of the patient in the context of the large aneurysm dimensions, significant thrombus burden, and the high rate of target lesion revascularization associated with the use of a covered stent.

Conflict of interest: Dr. Gerbaud is a consultant for Terumo Corporation. Dr. Osborn is a consultant for Abbott Vascular, Dynamed.

\section{References}

1. Vranckx P, Pirot L, Benit E. Giant left main coronary artery aneurysm in association with severe atherosclerotic coronary disease. Cathet Cardiovasc Diagn. 1997; 42(1): 54-57, indexed in Pubmed: 9286542.

2. Boyer N, Gupta R, Schevchuck A, et al. Coronary artery aneurysms in acute coronary syndrome: case series, review, and proposed management strategy. J Invasive Cardiol. 2014; 26(6): 283-290, indexed in Pubmed: 24907086.

3. Myler RK, Schechtmann NS, Rosenblum J, et al. Multiple coronary artery aneurysms in an adult associated with extensive thrombus formation resulting in acute myocardial infarction: successful treatment with intracoronary urokinase, intravenous heparin, and oral anticoagulation. Cathet Cardiovasc Diagn. 1991; 24(1): 51-54, indexed in Pubmed: 1913793.

4. Ibanez B, James S, Agewall S, et al. ESC Scientific Document Group. 2017 ESC Guidelines for the management of acute myocardial infarction in patients presenting with ST-segment elevation: The Task Force for the management of acute myocardial infarction in patients presenting with ST-segment elevation of the European Society of Cardiology (ESC). Eur Heart J. 2018; 39(2): 119-177, doi: 10.1093/ euheartj/ehx.393, indexed in Pubmed: 28886621.

5. Szalat A, Durst R, Cohen A, et al. Use of polytetrafluoroethylenecovered stent for treatment of coronary artery aneurysm. Catheter Cardiovasc Interv. 2005; 66(2): 203-208, doi: 10.1002/ccd.20448, indexed in Pubmed: 15977267.

6. Dutary J, Zakhem B, DE Lucas CB, et al. Treatment of a giant coronary artery aneurysm: intravascular ultrasound and optical coherence tomography findings. J Interv Cardiol. 2012; 25(1): 82-85, doi: 10.1111/j.1540-8183.2011.00659.x, indexed in Pubmed: 21599751.

7. Kim TH, Marfatia R, Lee J, et al. Giant coronary aneurysm management with Viabahn covered stent. Cardiovasc Revasc Med. 2017; 18(6S1): 56-59, doi: 10.1016/j.carrev.2017.03.019, indexed in Pubmed: 28483590.

8. Boi A, Sanna F, Rossi A, et al. Exclusion of a giant saphenous vein graft pseudo-aneurysm with a "double-layer bridging" technique. How should I treat multiple coronary aneurysms with severe stenoses. Cardiovasc Revasc Med. 2018; 92(7): E456-E460.

9. Warisawa T, Naganuma T, Nakamura S, et al. How should I treat multiple coronary aneurysms with severe stenoses? EuroIntervention. 2015; 11(7): 843-846, doi: 10.4244/EIJV11I7A171, indexed in Pubmed: 26603993.

10. Ferré Vallverdú M, Heredia Cambra T, Sanz Sánchez J, et al. An Unusual Cause of ST-Segment Elevation Myocardial Infarction. JACC Cardiovasc Interv. 2017; 10(9): 961-962, doi: 10.1016/j.jcin.2017.02.031, indexed in Pubmed: 28412253. 